\title{
Chemical Engineering Systems Stability
}

\section{Christo Boyadjiev}

Institute of Chemical Engineering, Bulgarian Academy of Sciences, Bulgaria

E-mail: chr.boyadjiev@gmail.com

\begin{abstract}
A theoretical analysis of the stability of the non-equilibrium chemical engineering systems is presented. A unified approach is proposed for the creation of the mathematical models of the systems that allows the determination of the velocities at which the systems move to their thermodynamic equilibriums and their use for mathematical analysis of systems velocities stability. For this purpose is used mathematical stability theory, evolution (autonomous) equations, bifurcation theory (stable focuses, stable cycles), parameter eigenvalues and eigenfunctions.
\end{abstract}

Keywords: power functions complex, stability theory, evolution equations, bifurcation theory, stable focuses, stable cycles, parameter eigenvalues

\section{Introduction}

Non-equilibrium chemical engineering systems are aggregations of physical, chemical and biological processes. They "move" to their thermodynamic equilibrium with a velocity that depends on the velocities of the individual processes. Non-equilibrium industrial systems are stable when two conditions are met: 1 . The velocity of movement towards their thermodynamic equilibrium is a constant; 2 . Capable of rapidly reaching their constant velocity at deviations, as a result of smooth change of external conditions.

The theoretical analysis of the stability of the non-equilibrium chemical engineering systems consists of 2 stages: 1 . Creation a mathematical model of the system, allowing the determination of the velocity at which the system moves to its thermodynamic equilibrium; 2 . Mathematical analysis of system velocity stability.

The first stage is different for individual systems, while the second stage is common to all industrial systems.

An unified approach will be proposed for the creation of a mathematical model of the system, which permits to be obtained the velocity of the system movement to its thermodynamic equilibrium and mathematical analysis of the system stability.

\section{Chemical engineering systems kinetics}

The kinetics of the chemical engineering systems depends on a set of variables. If the velocity of the industrial system is denoted by the values of these variables, the equation of the kinetic model of the industrial system will have the form:

$$
y=f\left(x_{1}, \ldots, x_{n}\right)
$$

This function is a mathematical structure that is retained when changed the measurement system of the variable, i.e. this mathematical structure is invariant with respect to similar transformations ${ }^{[1]}$ :

$$
\bar{x}_{i}=k_{i} x_{i}, \quad i=1, \ldots, n,
$$

i.e. $f$ is a homogeneous function:

$$
k y=f\left(k_{1} x_{1}, \ldots, k_{n} x_{n}\right) \varphi\left(k_{1}, \ldots, k_{n}\right) \cdot f\left(x_{1}, \ldots, x_{n}\right), \quad k=\varphi\left(k_{1}, \ldots, k_{n}\right) .
$$

From this it follows ${ }^{[1]}$ that it can be represented by a power functions complex:

Copyright (C2020 Christo Boyadjiev

DOI: https://doi.org/10.37256/sce.11202050.9-16

This is an open-access article distributed under a CC BY license

(Creative Commons Attribution 4.0 International License)

https://creativecommons.org/licenses/by/4.0/ 


$$
y=k x_{1}^{\alpha_{1}} x_{2}^{\alpha_{2}} \ldots x_{n}^{\alpha_{n}}
$$

where the parameters $k, \alpha_{1}, \ldots, \alpha_{n}$ are determined by experimental data of the chemical engineering system velocity. This allows to consider the velocity of the industrial system $y$ (phase velocity) as a point in a $n$ - dimension space, with coordinates $x_{1}, \ldots, x_{n}$ (phase space). Velocity projections on coordinate axes $x_{1}, \ldots, x_{n}$ are $\frac{d x_{i}}{d t}(i=1, \ldots, n)$ and satisfy the "evolution" law of the chemical engineering system:

$$
\frac{d x_{i}}{d t}=a_{i} y=a_{i} k x_{1}^{\alpha_{1}} \ldots x_{n}^{\alpha_{n}} ; \quad t=0, \quad x_{i}=x_{0 i} ; \quad i=1, \ldots, n,
$$

where $a_{1}, \ldots, a_{n}$ represent the ratios of variable $x_{1}, \ldots, x_{m}$ velocities and the industrial system velocity $y$.

The components of the phase velocity $\frac{d x_{i}}{d t}(i=1, \ldots, n)$ are the coordinates of the vector field at the same phase velocity and determine the velocity of motion of the chemical engineering system in the phase space. The points $x_{i}(t)(i=1, \ldots, n)$ represent a curve (phase trajectory) in the scalar phase space.

If we use the rule to differentiate a exponent function, the derivative of the velocity of the industrial system (4) over the time has the form:

$$
\frac{d y}{d t}=k \prod_{i=1}^{n}\left[x_{i}(t)\right]^{\alpha_{i}} \sum_{i=1}^{n}\left[\frac{\frac{d x_{i}}{d t}}{x_{i}(t)} \alpha_{i}\right]
$$

From this equation is possible to be obtained stability condition $\frac{d y}{d t}=0$ of the chemical engineering system:

$$
k \prod_{i=1}^{n}\left[x_{i}(t)\right]^{\alpha_{i}} \sum_{i=1}^{n}\left[\frac{\frac{d x_{i}}{d t}}{x_{i}(t)} \alpha_{i}\right]=0
$$

The solution of this set of equations allows the determination of the conditions for the stability of the chemical engineering system.

\section{Mathematical stability theory}

The velocity of the non-equilibrium industrial systems with which they "move" to their thermodynamic equilibrium is determined by the theory of the evolution equations ${ }^{[2-4]}$. Their capable of rapidly reaching their constant velocity at deviations, as a result of smooth change of external conditions, is determined by the theory of bifurcations ${ }^{[5]}$.

\subsection{Evolution equations}

Let consider the chemical engineering system velocity, which may be determined by the variables $x_{i}(i=1, \ldots, n)$ .This permits to consider the state of the system as a point in n-dimensional space with co-ordinates $x_{i}(i=1, \ldots, n)$ (a phase space).

The changing of the system velocity over the time is a vector in the $\mathrm{n}$-dimensional space. Its projections on coordinate axes $\frac{d x_{i}}{d t}(i=1, \ldots, n)$ satisfy the "evolution" law of the system: 


$$
\frac{d x_{i}}{d t}=X_{i}\left(x_{1}, \ldots, x_{n}, t\right), \quad x_{i}(0)=x_{i 0}, \quad i=1, \ldots, n .
$$

The evolution equations (8) for systems with laws independent of the time are termed autonomous equations:

$$
\frac{d x_{i}}{d t}=X_{i}\left(x_{1}, \ldots, x_{n}\right), \quad x_{i}(0)=x_{i 0}, \quad i=1, \ldots, n
$$

The components of the phase velocity $X_{i}(i, \ldots, n)$ are the co-ordinates of the vector field of the same phase velocity and determine the velocity of the system in the phase space. The points $x_{i}(t)(i, \ldots, n)$ represent a curve (a phase trajectory) in the scalar phase space (field).

For simplicity of explanation consider the autonomous equation

$$
\frac{d x}{d t}=X(x), \quad x(0)=x_{0}
$$

The system is stable when the system velocity does not change over the time:

$$
X(x)=0
$$

i.e.

$$
X(a)=0 .
$$

It follows from (12) that the point $x=a$ may be considered as a stationary point (the system velocity is constant over time). If $a=x_{0}$ it clear that

$$
x(t) \equiv a
$$

is a solution of (10), where $a$ is a singular point.

For simplicity will be considered the linear version of the equation (10) and its solution:

$$
\frac{d x}{d t}=\lambda x, \quad x(0)=x_{0}, \quad x=x_{0} \exp (\lambda t)
$$

It is follows from (14) that $x=0$ is a singular point, i.e. $x_{0}=0$ and the solution of (14) has the following features (see Fig. 1):

$$
\begin{aligned}
& \lambda<0, \quad \lim _{t \rightarrow \infty} x(t)=0, \quad \forall x_{0} ; \\
& \lambda=0, \quad x=x_{0}, \quad \forall x_{0} ;
\end{aligned}
$$




$$
\begin{array}{ll}
\lambda>0, & x=x_{0}, \text { at } t=0 ; \\
\lambda>0, & \lim _{t \rightarrow \infty} x(t) \rightarrow \infty, \quad \forall x_{0}>0 ; \\
\lambda>0, & \lim _{t \rightarrow \infty} x(t) \rightarrow-\infty, \quad \forall x_{0}<0 .
\end{array}
$$

The multiformity of the solution at $\lambda>0$ is not a result of its non-uniqueness, but this due to the solution instability with respect of the small perturbation of the initial condition $\left(x_{0}\right)$.
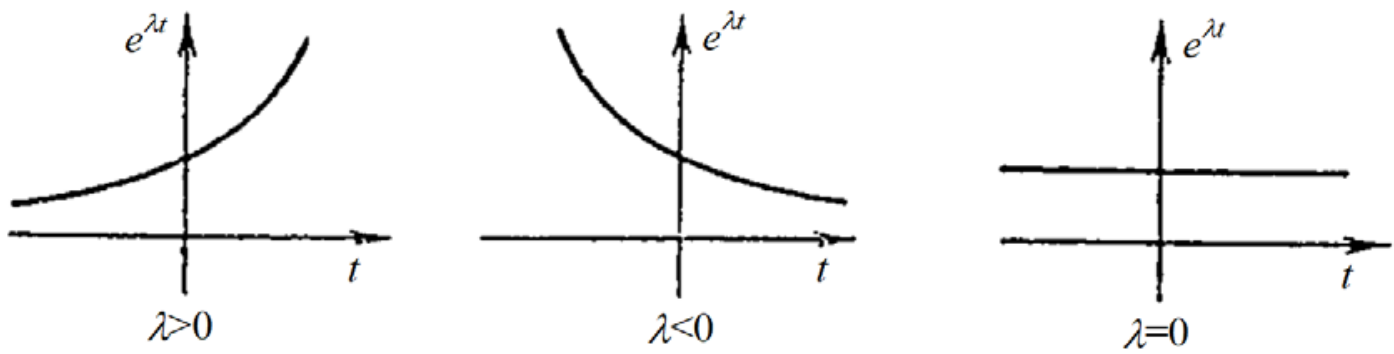

Figure 1. Solution of the equation (14)

The solution of the equation (14) leads to the following conclusions:

1. The solution (the process) is unstable at $\lambda>0$ and the small deviations of the initial state $x_{0} \neq 0$ lead to deviations of the solution $x=0$.

2. At $\lambda>0$ the solution is unstable for each $x_{0}$.

3. At $\lambda \leq 0$ the solution is approaching to the singular point , i.e. the stationary point become a focus of attraction of the solution (an attractor).

The linear equation (14), together with the conditions for the solution stability, are attractive because they give the basis of the kinetics models of many important processes (evolution of the organisms, nuclear processes, chemical reactions etc.) These features in the area of the real number $(R)$ become more interesting in the complex area $(C)$, where the equation (14) has the form:

$$
\frac{d z}{d t}=\lambda z, \quad z \in C, \quad \lambda \in C, \quad t \in R, \quad z(0)=z_{0}, \quad z(t)=z_{0} \exp (\lambda t)
$$

\subsection{Bifurcation theory}

The bifurcation theory ${ }^{[5]}$ is wide applied for investigations of jump reactions of systems as responses of smooth changes of the external conditions. For the real systems it has been developed recently as a theory of the catastrophes. Here, the bifurcation theory will be considered in two-dimensional phase space only.

For clarity of explanation, consider that a real evolutionary process occurring in the phase plane $(x, y)$ and the corresponding model is:

$$
\frac{d x}{d t}=X(x, y, \mu), \quad \frac{d y}{d t}=Y(x, y, \mu), \quad x(0)=x_{0}, \quad y(0)=y_{0}
$$

The system evolution in time is represented by the phase trajectory (the trajectory of the phase point) of the process

$$
F(x, y, \mu)=0
$$


where $x(t)$ and $y(t)$ in (20) are determined from the solution of (19). Depending on the form of the relationships for $X$ and $Y$ in (19), the parameter $\mu$ and the initial conditions $x_{0}$ and $y_{0}$ various phase trajectories are possible.

The variations of the parameter $\mu$ lead to several interesting cases of the solution of (19) shown in Fig. 2. The case shown in Fig. 2a corresponds to a periodic process that is attenuating with the time and approaching to a focus (a stationary state point). If other value of $\mu$ is chosen the process might be unstable and periodic (Fig. 2b). The stable periodic processes (limit cycles) have closed trajectories in the phase space (Fig. 2c). The change of the initial state $\left(y_{0}\right)$ of the stable processes leads to attenuating processes approaching a stable periodic state. (Fig. 2d).

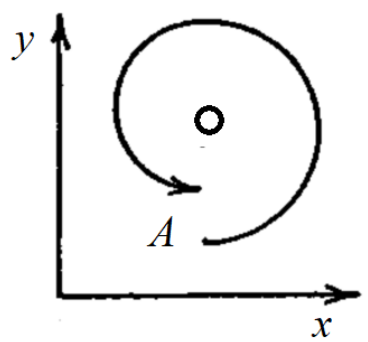

a

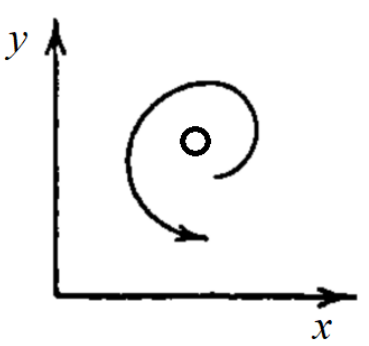

$\mathrm{b}$

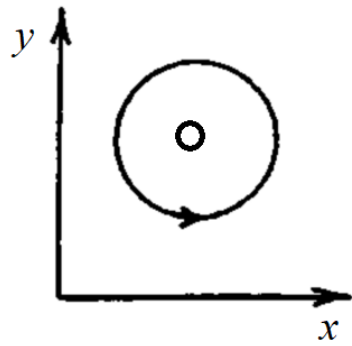

C

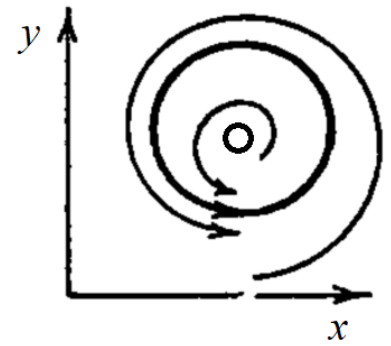

d

Figure 2. Phase trajectories

Figure 2 may be developed for more complicated cases (see Fig. 3). It is possible the existence of two limit cycles (periodic processes and solutions), where one of them (the internal) is stable if the initial conditions are in the entire internal area of the large cycle. The internal cycle attracts all the solutions, while the external cycle is unstable (Fig. 3a).

The variations of the parameter $\mu$ may lead to a junction of both cycles (Fig. 3b). The junction of an unstable and a stable cycle (as these in Fig. 3a) may lead to an abnormal limit cycle (Fig. 3b). In this case the solutions go from the initial conditions in internal area, approach the cycle and then due to small perturbations may go out of the cycle, so the process becomes unstable. The further changes of $\mu$ may lead to a situation when the limit cycle disappear and the process becomes unstable (Fig. 3c).

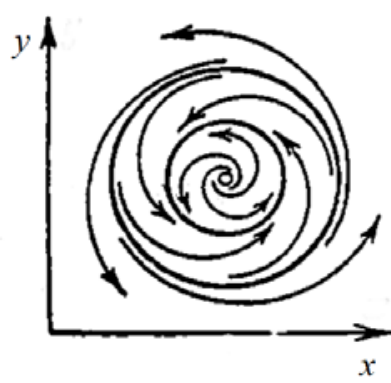

a

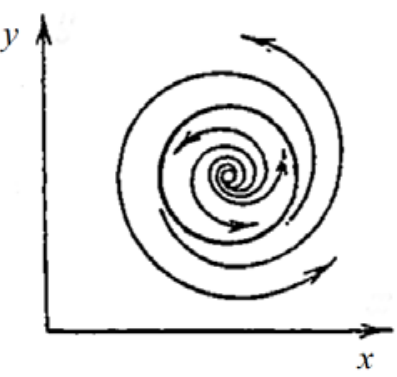

b

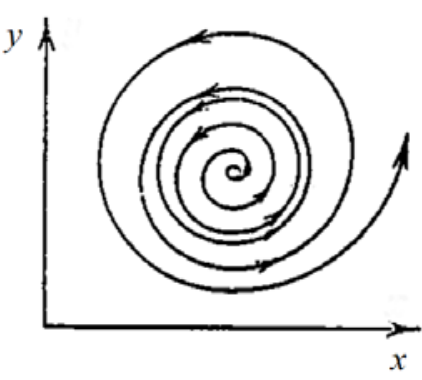

c

Figure 3. Limit cycles

The resulted obtained here show that the bifurcation theory considers qualitatively the changes of the movement of phase point as a result of a continuous variation of the model parameters. Parallel to the existence of stable points (focuses) there are stable cycles. They describe stationary periodic oscillations of the systems (self-oscillations). They differ from the free oscillations (of a pendulum for example) where the system does not interact with the environment as well as from the forced oscillations provoked by external periodic impacts.

The focuses and the limit cycles attracting the solution (the phase point) are termed attractors. 


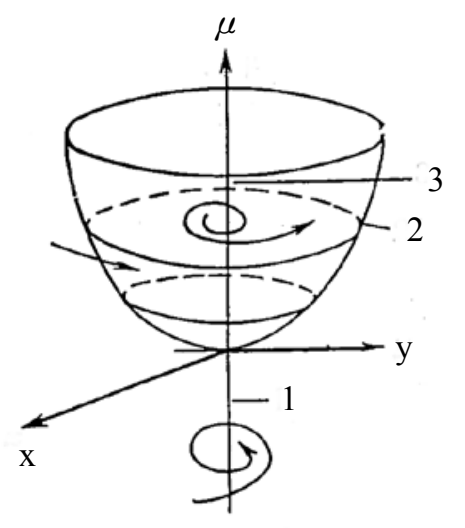

$$
\mu_{0}=0
$$

a

$\mathrm{X}$

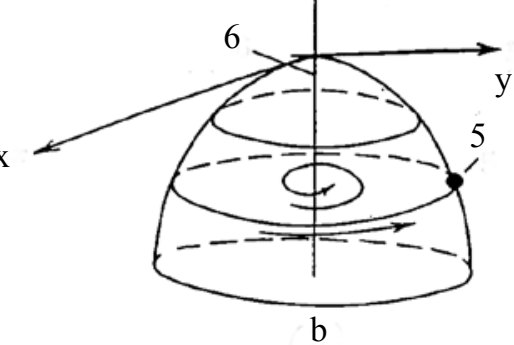

Figure 4. Bifurcation of cycle transitions from focuses

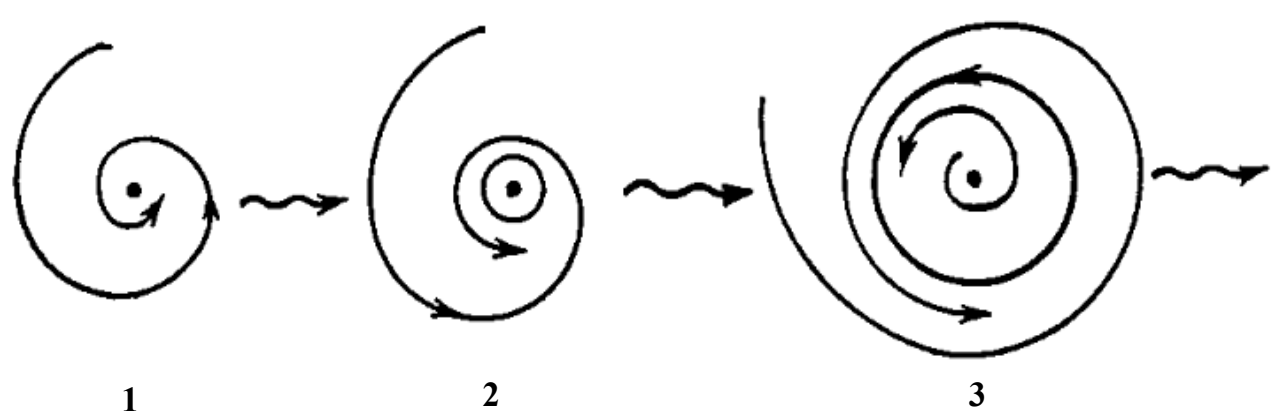

Figure 5. Mechanism of a transition from a focus toward a cycle

Figure 4 shows bifurcations of cycle transitions from focuses. The case (a) corresponds to a supercritical bifurcation (stable closed trajectories), while the case (b) presents a subcritical bifurcation (unstable and closed trajectories).

Further, Fig. 4a shows the mechanism of a transition from a stable point (focus) toward a stable orbit (cycle). This type of bifurcation is shown in Fig. 5. The stages of that transition are: 1) a stable point; 2) the occurrence of a closed trajectory; 3 ) an increase of the closed trajectory amplitude. This order leads to the existence of stable three-dimensional torus.

\subsection{Eigenvalue problems}

The presented analysis of the processes and the systems concerns its stabilities as functions of the model parameters. This requires a solution of differential equations with parameters. It is possible that the solution of the differential equation to exist at a given point (e.g. focus), only for a specific value of the parameter - "eigenvalue". For example, the secondorder ordinary differential equation, when the boundary conditions are presented at two different points, can have solution for a specific value of the parameter only, i.e. this leads to eigenvalue problems. The solution to this problem will be demonstrated in a first order linear ordinary homogeneous differential equation:

$$
y^{\prime}+[f(x)+\lambda g(x)] y=0,
$$

where $\lambda$ is a parameter and the solution must to satisfy the condition: 


$$
y(b)=\alpha y(a), \quad \alpha \neq 0 .
$$

The solution of (21) is well known

$$
y=C \exp \left[-\int_{a}^{x}(f+\lambda g) d x\right]
$$

The substitution of (23) in (22) shows that the condition (22) is satisfied, when $\lambda=\lambda_{0}$, only:

$$
\lambda_{0}=-\frac{\ln \alpha+\int_{a}^{b} f d x}{\int_{a}^{b} g d x}
$$

well known as an eigenvalue. The substitution of (24) in (23) leads to an eigenfunction. Thus, for example at $f \equiv 0$ and $g \equiv 1$ it follows directly:

$$
\lambda_{0}=\frac{\ln \alpha}{a-b}, \quad y=C \exp \left[-\frac{\ln \alpha(x-a)}{a-b}\right]
$$

It is well demonstrated in the differential equation theory ${ }^{[6]}$ that if $\int_{a}^{b} g d x \neq 0$ there is an infinite set of eigenvalues:

$$
\lambda_{k}=\lambda_{0}+\frac{2 k \pi i}{\int_{a}^{b} g d x}, \quad k=0, \pm 1, \pm 2, \ldots
$$

The results obtained are used ${ }^{[7]}$ for the theoretical analysis of the hydrodynamics stability in systems with non-linear mass transfer.

\section{Conclusions}

The presented theoretical analysis shows, that the stability of the non-equilibrium chemical engineering systems (aggregations of physical, chemical and biological processes) is possible to be analyzed by the creation a mathematical model of the system, the determination of the velocity at which the system moves to its thermodynamic equilibrium and mathematical analysis of system velocity stability.

A unified approach is proposed for the creation of the mathematical models of the systems that allows the determination of the velocities at which the systems move to their thermodynamic equilibriums and their use for mathematical analysis of systems velocities stability. For this purpose is used mathematical stability theory, evolution (autonomous) equations, bifurcation theory (stable focuses, stable cycles), parameter eigenvalues and eigenfunctions.

\section{References}

[1] Chr. Boyadjiev. Theoretical Chemical Engineering: Modeling and simulation. Berlin Heidelberg: Springer-Verlag; 2010. p. 594. 
[2] V. I. Arnold. Ordinarily Differential Equations. Nauka, Moskow; 1984 (in Russian).

[3] M. Braun. Differential Equations and their Applications. New York: Springer-Verlag; 1978.

[4] D. Zwillinger. Handbook of Differential Equations (ed. II). Boston: Acad. Press. Inc.; 1957.

[5] J. E. Marsden, M. McCracken. The Hopf Bifurcation and its Application. New York: Springer-Verlag; 1976.

[6] E. Kamke. Differentialgleichungen, Leipzig, 1959.

[7] Chr. Boyadjiev, V. N. Babak. Non-Linear Mass Transfer and Hydrodynamic Stability. Amsterdam: Elsevier; 2000. 